\title{
Explanation of Distance, Kinematic and Isometry to High School Students from Different Perspective
}

\author{
Abdullah Kurudirek (Corresponding author) \\ Department of Mathematics Education, Ishik University \\ 100 m St. \& Mosul Road, Erbil-Iraq
}

Tel: 964-750-706-5024_E-mail: drcebir@hotmail.com

\begin{abstract}
Huseyin Akca
Department of Mathematics Education, Ishik University

100 m St. \& Mosul Road, Erbil-Iraq
\end{abstract}

Tel: 964-750-835-7525Ｅ-mail: huseyinakcha@gmail.com

Received: December 2, 2014 Accepted: January 26, 2015 Published: February 2, 2015

doi:10.5296/ire.v3i1.7036 URL: http://dx.doi.org/10.5296/ire.v3i1.7036

\begin{abstract}
In this paper we try to show that the idea of distance in geometry is larger than the idea of metric in mathematics, and by this paper students will be able to learn and apply the proof of the idea that we mentioned above. The idea of this paper was thought during an international conference on 20 May 2013 in Uzbekistan.
\end{abstract}

Keywords: distance, euclidean, galilean, minkowskian, metric, limited point geometry, kinematic, isometry

\section{Introduction}

As it is well known, Geometry is a subject with which we are all familiar from high school and which investigates properties of figures in plane or in space. We know that the study of geometry goes back at least four thousand years, as far back as the Babylonians. Their geometry was empirical, and limited to those properties physically observable. The $19^{\text {th }}$ century was a period of rapid development in geometry. Modern definition of geometry was given by Thurston (1997), and this definition will be our guide. 


\section{Macrothink Institute $^{\text {TM }}$}

Studying geometry is not just learning the definitions or the attributes of geometrical concepts, but also to have the ability of analyzing the properties of geometric topics, shapes and develop mathematical arguments about geometric relationships. Geometry is not only earth measurement, as it is mentioned in ancient Greek, it may be true but not for all the planes. To be successful in learning Geometry, the understanding of geometrical concepts is essential. Especially, the distance idea is very important in Geometry. Studying geometry is an important component of learning mathematics because it allows students to analyze and interpret the world they live in as well as equip them with tools they can apply in other areas of mathematics. Therefore, students need to develop an understanding of geometric concepts as well as gaining adequate geometry related skills. In this project, analysis of the development of geometric skills about distance, kinematic and isometry are explored in different geometrical planes.

\section{Distance}

The idea of distance is of course a powerful one in geometry. First, let's meet the distance in the Euclidean plane denoted by $R_{2}$ or $E_{2}$. Let $R_{2}$ plane and $O x y$ decart coordinate system on this plane are given. In this case, the distance between the points $A\left(x_{1}, y_{1}\right)$ and $B\left(x_{2}, y_{2}\right)$ is calculated as $d(A, B)=\sqrt{\left(x_{2}-x_{1}\right)^{2}+\left(y_{2}-y_{1}\right)^{2}}$. And a metric is required to satisfy the following conditions.

(1) $\rho(x, y)=k \geq 0, k=0$ where $x=y$

(non-negativity)

(2) $\rho(x, y)=\rho(y, x)$

(symmetry)

(3) $\rho(x, y) \leq \rho(x, z)+\rho(z, y)$

(triangle inequality)

\subsection{Example 1}

For the distance between two points, $d(A, B)=\sqrt{\left(x_{2}-x_{1}\right)^{2}+\left(y_{2}-y_{1}\right)^{2}}$ we can easily see that the metric conditions satisfy. Now, let's show that the idea of distance is larger than the idea of metric.

Take $d(x, y)$ as a distance and it satisfies only the following two conditions.

(1) $d(x, y)=k$ where $k$ is a $\{-, 0,+\}$ quantity

(2) $d(x, y)=d(y, x)$

(symmetry)

As it is seen, the conditions of distance are less than the conditions of metric. If we include triangle inequality and non-negativity conditions then the distance will be metric. Let's think the distance between two cities Erbil and Sulaimania in Iraq. $d(e, s)=203 \mathrm{~km}$ this is a quantity of distance. Just like that, we can also think the distance as duration of the trip from one city to another so $d(e, s)=3 h$ (by bus), $d(e, s)=1 h$ (by plane). On the other hand, the distance can be thought as the cost of trip so $d(e, s)=20 \$$ (by bus), $d(e, s)=100 \$$ (by plane), $d(e, s)=10$ (by own car, only petrol costs), $d(e, s)=0 \$$ (the distance is taken by a friend's car which goes to the same direction).

It is obviously seen that the distance can be in all sorts of quantity like time, cost,... etc. Apart from, what about the distance concept for the hostess? If we think the distances which are explained above as positive quantities then, for hostess distance may be thought as a 
negative quantity!

In Minkowskian plane, $d(A, B)=\sqrt{\left(x_{2}-x_{1}\right)^{2}-\left(y_{2}-y_{1}\right)^{2}}$ provides the distance conditions, but it is not metric.

(1) If $\left(x_{2}-x_{1}\right)^{2}-\left(y_{2}-y_{1}\right)^{2}>0$ then distance is real

(2) If $\left(x_{2}-x_{1}\right)^{2}-\left(y_{2}-y_{1}\right)^{2}=0$ then the points can't be coincide

(3) If $\left(x_{2}-x_{1}\right)^{2}-\left(y_{2}-y_{1}\right)^{2}<0 \quad$ then distance is imaginary

\subsection{Example 2}

Find the distance between the points $A(3,5)$ and $B(11,13)$.

In Minkowskian plane, it is $d=\sqrt{(11-3)^{2}-(13-5)^{2}}=\sqrt{(8)^{2}-(8)^{2}}=0$. As you see $d=0$ but $A \neq B$ (they are not same points, not coincide) even it doesn't satisfy the first condition of metric, it is a distance.

In Galilean plane, distance is calculated between the two points $A\left(x_{1}, y_{1}\right)$ and $B\left(x_{2}, y_{2}\right)$ (Figure 1) as, $d_{1}=\left|x_{2}-x_{1}\right|$, if $d_{1}=0$ then $d_{2}=\left|y_{2}-y_{1}\right|$. So, we are taking the distance as projection, if $d_{1}=0$ and $d_{2}=0$ then $A=B$ (the points are coincide).

It provides distance conditions but not metric, since the third condition of metric (triangle inequality) doesn't satisfy as it is seen in the following Figure 2.

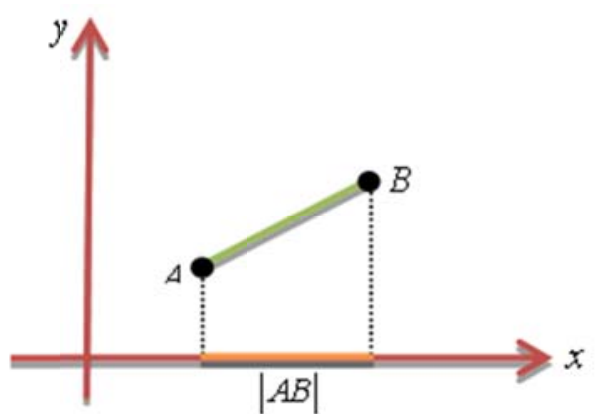

Figure 1. Distance in Galilean Plane

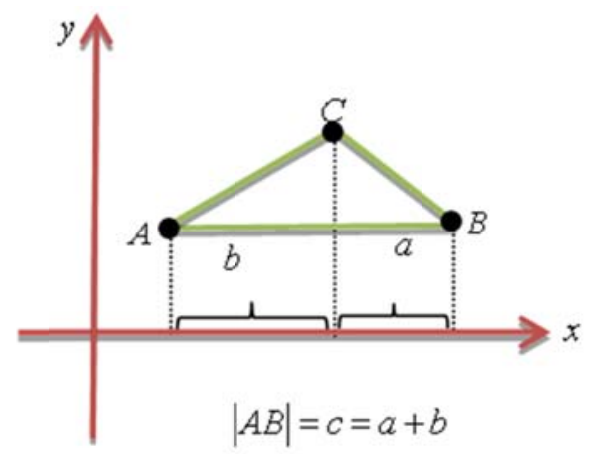

Figure 2. Triangle inequality in Galilean Plane

\section{Kinematic and Isometry}

Kinematic is a branch of classical mechanics which describes the motion of points, bodies and systems of bodies without consideration of the causes of motion. What about isometry? An isometry is a transformation in which the original figure and its image are congruent. If we talk more about isometry, we may say; isometry is invariant with respect to distance. That is, in an isometry, the distance between any two points in the original figure is the same as the distance between their corresponding images in the transformed figure (image). Reflections, rotations, translations are isometries. Dilation is not an isometry. Simply, kinematics can be shown in the following ways: 
When we shift the origin $O$ to $O^{\prime}$ the kinematic may be got as $\left\{\begin{array}{l}x^{\prime}=x+a \\ y^{\prime}=y+b\end{array}\right.$, and direction of the coordinate axis are same (Figure 3).

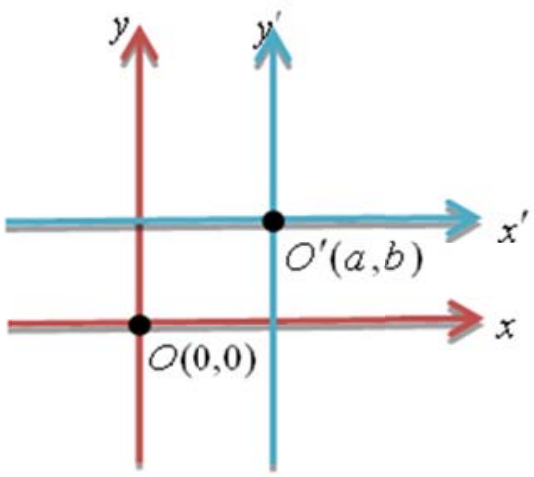

Figure 3. Shifting Origin

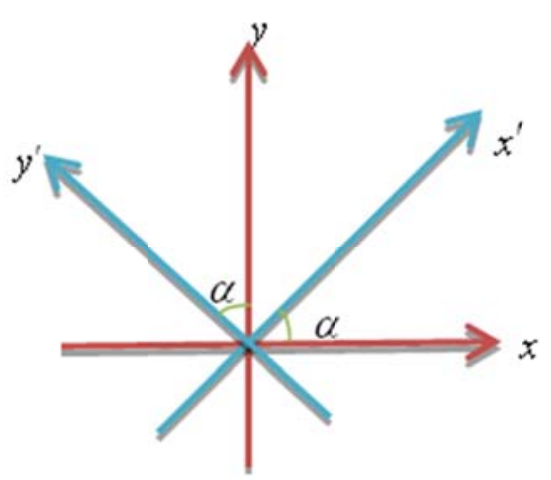

Figure 4. Rotation of Axes

When the direction of the arrows are rotated by $\alpha$, the kinematic may be got as $\left\{\begin{array}{l}x^{\prime}=x \cos \alpha-y \sin \alpha \\ y^{\prime}=x \sin \alpha+y \cos \alpha\end{array}\right.$ (Figure 4).

The advantage of shifting and rotating of coordinate shows the equation in simple form. This rotating and shifting is called kinematic(motion), and this motion preserves the distance between two points. According to the different planes, the distances and kinematics can be expressed as follows.

\subsection{Galilean Plane}

The distance between two points in Galilean plane is $d_{1}=\left|x_{2}-x_{1}\right|$, if $d_{1}=0$ then $d_{2}=\left|y_{2}-y_{1}\right|$, and the kinematic which preserves the distance is $f:\left\{\begin{array}{l}x^{\prime}=x+a \\ y^{\prime}=h x+y+b\end{array}\right.$.

\subsection{Minkowskian Plane}

The distance between two points in Minkowskian plane is $\quad d(A, B)=\sqrt{\left(x_{2}-x_{1}\right)^{2}-\left(y_{2}-y_{1}\right)^{2}}$, and the kinematic which preserves the distance is $f:\left\{\begin{array}{l}x^{\prime}=x \cosh \alpha+y \sinh \alpha+a \\ y^{\prime}=x \sin h \alpha+y \cosh \alpha+b\end{array}\right.$.

\subsection{Euclidean Plane}

The distance between two points in Euclidean plane is $d(A, B)=\sqrt{\left(x_{2}-x_{1}\right)^{2}+\left(y_{2}-y_{1}\right)^{2}}$, and the kinematic which preserves the distance is $f:\left\{\begin{array}{l}x^{\prime}=x \cos \alpha-y \sin \alpha+a \\ y^{\prime}=x \sin \alpha+y \cos \alpha+b\end{array}\right.$.

\subsection{Example}

Find the new vertices and area of the right angled triangle $\triangle A O B$ whose vertices are $O(0,0), \mathrm{A}(4,0), \mathrm{B}(0,4)$ after rotating by $\alpha=60^{\circ}$ and shifting $a=1, b=1$ unit.

Solution: After doing necessary algebraic operations, we get the vertices of the new right 
angled triangle $\Delta A^{\prime} O^{\prime} B^{\prime}$ as follows:

$A^{\prime}:\left\{\begin{array}{l}x^{\prime}=4 \cos 60^{\circ}-0 \sin 60^{\circ}+1=3 \\ y^{\prime}=4 \sin 60^{\circ}+0 \cos 60^{\circ}+1=1+2 \sqrt{3}\end{array}, \quad B^{\prime}:\left\{\begin{array}{l}x^{\prime}=0 \cos 60^{\circ}-4 \sin 60^{\circ}+1=1-2 \sqrt{3} \\ y^{\prime}=0 \sin 60^{\circ}+4 \cos 60^{\circ}+1=3\end{array} \quad\right.\right.$ and $\quad O^{\prime}:\left\{\begin{array}{l}x^{\prime}=0-0+1=1 \\ y^{\prime}=0+0+1=1\end{array}\right.$

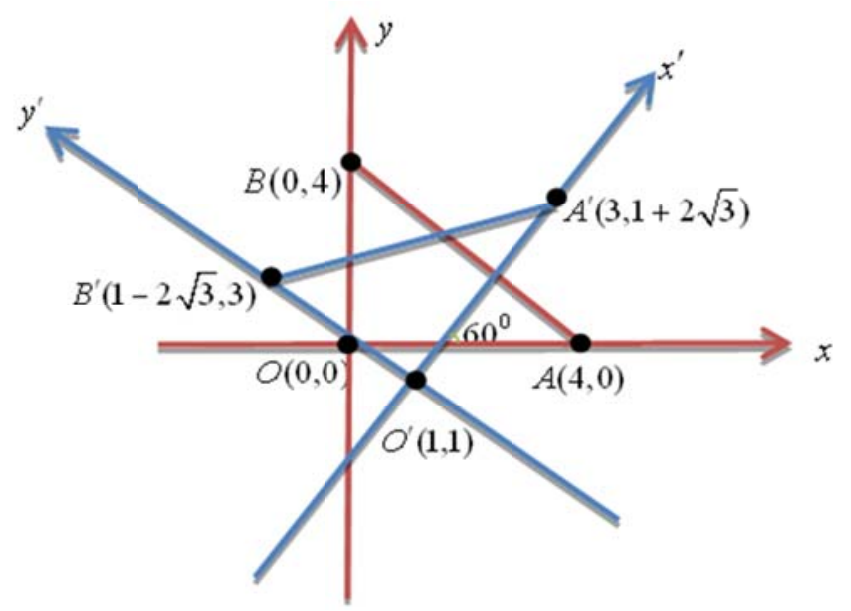

Figure 5. Example

Now, let's compare some properties of these two right angled triangles. For example $|O A|=4$, $|O B|=4,|A B|=4 \sqrt{2}$ and $S_{A O B}=\frac{1}{2}|O A||O B|=\frac{1}{2} 4.4=8$ unit square.

$\left|O^{\prime} A^{\prime}\right|=\sqrt{(1+2 \sqrt{3}-1)^{2}+(1-3)^{2}}=\sqrt{12+4}=\sqrt{16}=4, \quad\left|O^{\prime} B^{\prime}\right|=\sqrt{(1+2 \sqrt{3}-1)^{2}+(1-3)^{2}}=\sqrt{12+4}=\sqrt{16}=4$, $\left|A^{\prime} B^{\prime}\right|=\sqrt{(-2 \sqrt{3}+1-3)^{2}+(3-2 \sqrt{3}-1)^{2}}=\sqrt{12+8 \sqrt{3}+4+4-8 \sqrt{3}+12}=\sqrt{32}=4 \sqrt{2}$ and $S_{A^{\prime} O^{\prime} B^{\prime}}=\frac{1}{2}\left|O^{\prime} A^{\prime}\right|\left|O^{\prime} B^{\prime}\right|=\frac{1}{2} 4.4=8$ unit square.

\section{Limited Point Geometry}

So far, we have studied about plane geometry. Now, we can see the same ideas in limited point geometry in which we don't need plane.

\section{0}

Let's define the distance in this geometry as the shortest path which is taken

000 by the number of the points, and it allows $\rightarrow$ horizontal, $\downarrow$ vertical and diagonal, as one unit.

000

$$
a_{11} \quad a_{12} \quad a_{13}
$$

e.g. $\quad d\left(a_{11}, a_{23}\right)=2$ (passing through $\left.\quad a_{11} \rightarrow a_{22} \rightarrow a_{23}\right)$ and $d\left(a_{21}, a_{33}\right)=2 \quad a_{21} \quad a_{22} \quad a_{23}$

$$
\begin{array}{lll}
a_{31} & a_{32} & a_{33}
\end{array}
$$




\section{Macrothink}

Totally, we have $s=\frac{1}{2} C_{9}^{2}=\frac{1}{2} \cdot \frac{9 \cdot 8.7 !}{7 ! .2}=18$ different distances. Let us explain the distance on different examples as follows:

(1) Circle is the curve traced out by a point that moves, so that its distance from a given point is constant (Figure 6). So $a_{22}$ is at the center and the other points are equidistant to $a_{22}$.

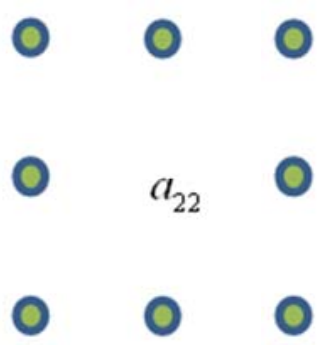

Figure 6. Circle in Limited point geometry

(2) What about square? The answer is clear; again we get the same Figure 6.

(3) What about line in this geometry? As it is mentioned above, we define line as the shortest distance which joins two points.

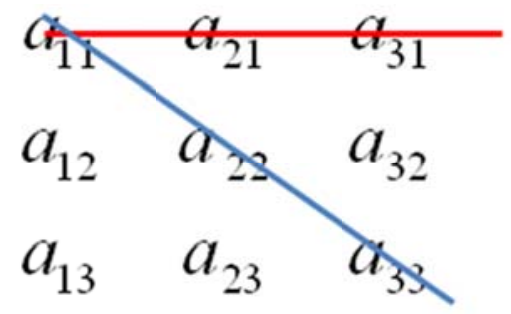

Figure 7. Lines

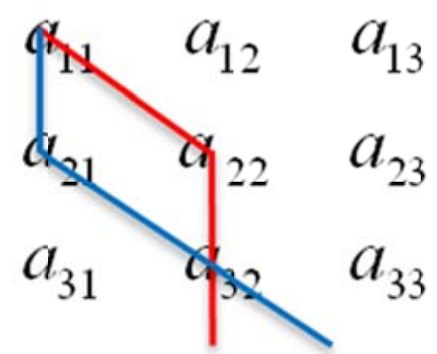

Figure 8. Lines in Limited point geometry

In Figure 7, the red line passes through $a_{11}$ and $a_{21}$, the blue one passes through $a_{11}$ and $a_{22}$. These lines in Figure 8 pass through $a_{11}$ and $a_{32}$. The Euclidean's axiom is broken in this geometry, because it says "just a line passes through two points"! Now, let's try to show a triangle in this geometry.

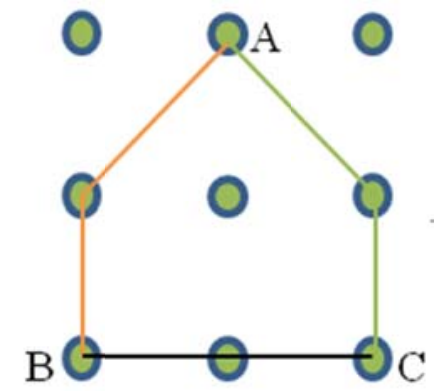

Figure 9. Triangle in Limited point geometry

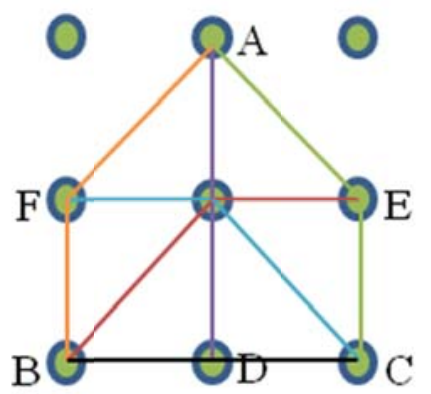

Figure 10. Basic elements of the triangle 


\section{Macrothink}

As shown in Figure 9, we can see different triangles in that way, and triangle inequality can be seen easily i.e. $B C \leq A B+A C$. Figure 10 indicates the distances: $\mathrm{AD}$ median (in $\mathrm{ABC}$ equilateral triangle), $\mathrm{BE}$ median (BE is a straight line), and $\mathrm{CF}$ median (CF is a straight line). We have defined distance, now we need the kinematic which preserves this distance, and it can be shown as follows:

Diagonally

$$
\begin{array}{lll}
a_{11} & a_{21} & a_{31} \\
a_{12} & a_{22} & a_{32} \\
a_{13} & a_{23} & a_{33}
\end{array}
$$

Horizontally

$\begin{array}{lll}a_{31} & a_{32} & a_{33} \\ a_{21} & a_{22} & a_{23} \\ a_{11} & a_{12} & a_{13}\end{array}$

Vertically

$\begin{array}{lll}a_{13} & a_{12} & a_{11} \\ a_{23} & a_{22} & a_{21} \\ a_{33} & a_{32} & a_{31}\end{array}$

We may do the same operations by using 16 points, and for this one we have $s=\frac{1}{2} C_{16}^{2}=\frac{1}{2} \cdot \frac{16 \cdot 15}{2}=60$ different distances.

Finally, we may use a chess board (64 points!) and on the chess board by chess pieces some of the kinematics will be as follows:

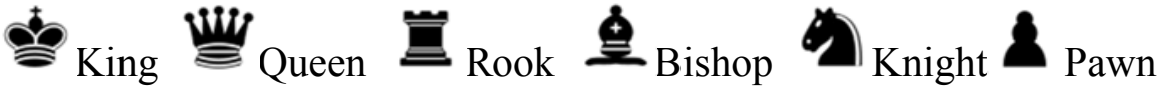

1) According to King $\quad: \rightarrow$ horizontal, $\downarrow$ vertical and diagonal.

2) According to Rook $\quad: \rightarrow$ horizontal, and $\downarrow$ vertical

3) According to Knight $\quad: \neg$ and $」$ like letter $L$.

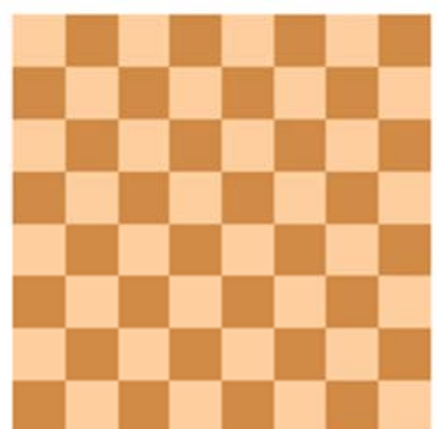

Figure 11. Chess board

By using the same definitions; triangle, square, and circle can be shown. Attention to the figures which can't be familiar with the one we have studied before!

\section{Conclusion}

The aim of the paper is to make the teachers and high school students become aware of distance, kinematic and isometry topics and provide them a new perspective. In that regard, the results of the study revealed may serve well as a source for both learning and teaching some geometric concepts. We can complete our paper by modern definition of geometry which is given by W.P Thurston, as taking $d(x, y)$ distance such that $d(x, y)=d(f(x), f(y))$ and $d(x, y)=d^{*}\left(x^{*}, y^{*}\right)$. If this condition provides $d(x, y)=d^{*}\left(x^{*}, y^{*}\right)=d(f(x), f(y))$ then it is called isometry, and denoted by $Y=$ Isom $X$. The science that teaches the properties of the elements of the sum $(X, \operatorname{Isom} X)$ is called geometry. High school students visually recognize geometric topics and figures by their global appearance. They recognize circles, triangles, squares, ..., by their shapes, now they will explicitly identify the appearance from another perspective. 


\section{Macrothink Institute ${ }^{\mathrm{TM}}$}

And also, they will be able to start analyzing the properties of geometric topics, figures and learn the appropriate terminology to describe them, after this article, perhaps they will search interrelate technical terminology to describe figures or properties of figures.

\section{References}

Artıkbayev, A., \& Sokolov, D. D. (1991). Geometry as a whole in a flat space-time. Tashkent. Ed. "Fan".

Artıkbayev, A., Kurudirek, A., \& Akça, H. (2013). Occurrence of Galilean Geometry. Applied $\begin{array}{lll}\text { and Computational } \quad \text { Mathematics, } & \text { 2(5), }\end{array}$ http://dx.doi.org/10.11648/j.acm.20130205.11

Akar, M., Yüce, S., \& Kuruoğlu, N. (2013). One-Parameter Motion on the Galilean Plane. International Electronic Journal of Geometry, 6(1), 79-88.

Bartlett, P. (2012). Latin Squares and Geometry. Lecture notes math camp.

Kurudirek, A., Akça, H., \& Erdoğan, M. (2013). On Geometries in Affine Plane. Applied and Computational Mathematics, 2(6), 127-129. http://dx.doi.org/10.11648/j.acm.20130206.13

Ортиқбоев, А. (2004). Геометрия - қадимий фанга замонавий назар. Физика, математика, информатика журнали.

Thurston, W. P. (1997). Three-Dimensional Geometry and Topology, Volume 1 (Silvio Levy Ed). Princeton University Press.

Yaglom, I. M. (1979). A Simple Non-Euclidean Geometry and Its Physical Basis. Springer-Verlag New York Inc.

\section{Copyright Disclaimer}

Copyright reserved by the authors.

This article is an open-access article distributed under the terms and conditions of the Creative Commons Attribution license (http://creativecommons.org/licenses/by/3.0/). 